\title{
Fast Fourier Transform of Multi-Assets Options under Economic Recession Induced Uncertainties
}

\author{
Philip Ajibola Bankole, Olabisi 0. Ugbebor \\ Department of Mathematics, University of Ibadan, Ibadan, Nigeria \\ Email: pa.bankole@yahoo.com, ugbebor1@yahoo.com
}

How to cite this paper: Bankole, P.A. and Ugbebor, O.O. (2019) Fast Fourier Transform of Multi-Assets Options under Economic Recession Induced Uncertainties. American Journal of Computational Mathematics, 9, 143-157.

https://doi.org/10.4236/ajcm.2019.93011

Received: July 1, 2019

Accepted: August 27, 2019

Published: August 30, 2019

Copyright $\odot 2019$ by author(s) and Scientific Research Publishing Inc. This work is licensed under the Creative Commons Attribution International License (CC BY 4.0).

http://creativecommons.org/licenses/by/4.0/

\begin{abstract}
A Fast Fourier transform approach has been presented by Carr \& Madan (2009) on a single underlying asset. In this current research paper, we present fast Fourier transform algorithm for the valuation of Multi-asset Options under Economic Recession Induced Uncertainties. The issue of multi-dimension in both finite and infinite case of Options is part of the focus of this research. The notion of economic recession was incorporated. An intuition behind the introduction of recession induced volatility uncertainty is revealed by huge volatility variation during the period of economic recession compared to the period of recession-free. Nigeria economic recession outbreak in 2016 and its effects on the uncertainty of the payoffs of Nigeria Stocks Exchange (NSE) among other investments was among the motivating factors for proposing economic recession induced volatility in options pricing. The application of the proposed Fast Fourier Transform algorithm in handling multi-assets options was shown. A new result on options pricing was achieved and capable of yielding efficient option prices during and out of recession. Numerical results were presented on assets in 3-dimensions as an illustration taking Black Scholes prices as a bench mark for method effectiveness comparison. The key findings of this research paper among other crucial contributions could be seen in computational procedure of options valuation in multi-dimensions and uncertainties in options payoffs under the exposure of economic recession.
\end{abstract}

\section{Keywords}

Fast Fourier Transform (FFT), Multi-Assets, Finite and Infinite Dimension of Assets, Economic Recession, Volatility Change, European Options

\section{Introduction}

In the history of options valuation, the work of Black-Scholes [1] (1973) cannot 
be forgotten no matter the shortcomings we may find in their work such as the issue of constant values assumed by the volatility and interest rates. Divers financial assets are traded in financial market. Some are risky assets while some are riskfree. Multi-asset options generally are a set of options whose pay-offs are governed by more than one underlying assets. A typical example of multi-asset option is a basket option. Basket option is based on two or more underlying assets or stocks. There is European type basket call or put option whereby the overall value of a basket of assets plays the role of the price of the underlying asset in single-asset European. Likewise, we have American style basket options as well. Other types of multi-assets options include: spread option, exchange options, Quanto options, Rainbow options, etc. Various types of multi-asset options could be described based on their payoff function. Beom, J. K. et al. (2013) [2] presents a simple numerical approach for American put options valuation.

The focus of most researchers is limited to two dimensions in the valuation of multi-asset options. Some authors such as Yuwei Chen [3] gave their formulation in terms of Black Scholes Partial Differential Equation (PDE) and considered numerical method for obtaining the prices. The authors, Carol Alexander et al., (2009) [4] did not relent in their effort as they presented an analytic approximation for Multi-asset Options Pricing but their approach was different from the one we gave here. In recent time, an existence result of solutions for a multi-dimensional stochastic control problem with singular state constraint was presented by Ulrich Horst and Xiaonyu Xia, (2018) [5]. The problem at hand is not centered on the specialty of the type of multi-asset but how to handle the nature of multi-assets amidst other conditional forces of uncertainty and dimensional challenges in the valuation process. According to Yuwei Chen [3], most multi-asset European options and all American options have no analytical formula giving their prices, therefore, numerical methods are employed to approximate such prices.

We consider challenges of uncertainty posed by instability of economic factors on the payoff of assets. The keen economic factor to us is recession. We consider volatility variation imposed on options prices during economic recession which we termed economic recession induced volatility in addition to other volatility uncertainties of options price from other sources. Fast Fourier Transform algorithm is used in our formulation. Many authors have worked on Fourier transform of some options valuation such as ([6] [7] [8] [9]) among others. We gave a framework for extending Carr \& Madan [6] Fast Fourier Transform approach of valuating an option on a single underlying asset to valuation of multi-assets options. Our major contribution is seen in valuation of multi-assets option in both finite and infinite dimensions and incorporation of economic recession factor on the volatility of assets during the state of economic recession.

The rest part of this paper is organized as follows. In Section 2, we gave preliminaries on Fast Fourier Transform. Section 3 is the Main result, which consists of subsections Fast Fourier Transform of multi-assets in finite dimension 
and Fast Fourier Transform of multi-assets in infinite dimension. Section 4 is the application on three assets in 3-dimensional case while Section 5 shows $\mathrm{Nu}$ merical results on multi-assets in 3-dimensions. The last section is the conclusion part.

\section{Preliminaries}

\subsection{Some Definitions on Fourier Transform}

Definition 1: Let $f: R \rightarrow R$ be a real-value function for $x \in R$, then the Fourier transform of $f$ is defined as

$$
\mathcal{F}(f(x) ; w)=\hat{f}(w)=\int_{-\infty}^{\infty} f(x) \mathrm{e}^{-i \omega x} \mathrm{~d} x x^{\varkappa}, \omega \in R,
$$

where $i=\sqrt{-1}$ and $\omega$ is a parameter. We can recover $f(x)$ from $f(\omega)$ by the inverse Fourier transform.

Definition 2: The inverse Fourier transform of the real valued function $f(x)$ is defined by

$$
\mathcal{F}^{-1}(\hat{f}(\omega) ; x)=f(x)=\frac{1}{2 \pi} \int_{-\infty}^{\infty} \hat{f}(w) \mathrm{e}^{i \omega x} \mathrm{~d} \omega, \quad x \in R
$$

which belongs to $L^{2}$-spaces.

Some certain axioms on Fourier transform exist in literature. Among such axioms are: convolution axiom, Translation axiom, Linearity axiom, Differentiation axiom, Symmetric axiom etc.

\subsection{Some Axioms of Fourier Transform}

- Convolution axiom: This property is considered very crucial as convolution of functions is mapped into multiplication in Fourier space.

Let $f, g \in L^{2}$-space.

Then their convolution is defined by

$$
(f \star g)(x)=\int_{-\infty}^{\infty} f(x-y) g(y) \mathrm{d} y=\int_{-\infty}^{\infty} f(y) g(x-y) \mathrm{d} y
$$

The Fourier transform of the convolution of the functions $f$ and $g$ with convolution operator $\star$ is given by

$$
\mathcal{F}((f \star g) ; \omega)=\int_{-\infty}^{\infty}[f(x-y) g(y) \mathrm{d} y] \mathrm{e}^{-i \omega x} \mathrm{~d} x=\hat{f}(\omega) \hat{g}(\omega)
$$

The proof of Equation (4) is given in what follows.

\section{Proof:}

$$
\begin{aligned}
& \mathcal{F}((f \star g) ; \omega)=\int_{-\infty}^{\infty}\left[\int_{-\infty}^{\infty} f(x-y) g(y) \mathrm{d} y\right] \mathrm{e}^{-i \omega x} \mathrm{~d} x \\
& \mathcal{F}((f \star g) ; \omega)=\int_{-\infty}^{\infty}\left[\int_{-\infty}^{\infty} f(x-y) g(y) \mathrm{e}^{-i \omega x} \mathrm{~d} y\right] \mathrm{d} x \\
& \mathcal{F}((f \star g) ; \omega)=\int_{-\infty}^{\infty}\left[\int_{-\infty}^{\infty} f(x-y) g(y) \mathrm{e}^{-i \omega x} \mathrm{~d} x\right] \mathrm{d} y
\end{aligned}
$$




$$
\mathcal{F}((f \star g) ; \omega)=\int_{-\infty}^{\infty} \nvdash(y)\left[\int_{-\infty}^{\infty} f(x-y) \mathrm{e}^{-i \omega x} \mathrm{~d} x\right] \mathrm{d} y
$$

To this end, one will have

$$
\begin{gathered}
\mathcal{F}((f \star g) ; \omega)=\int_{-\infty}^{\infty} g(y) \mathrm{e}^{-i \omega y}\left[\int_{-\infty}^{\infty} f(\zeta) \mathrm{e}^{-i \omega(\zeta+y)} \mathrm{d} \zeta\right] \mathrm{d} y \\
\mathcal{F}((f \star g) ; \omega)=\int_{-\infty}^{\infty} g(y) \mathrm{e}^{-i \omega(y)}\left[\int_{-\infty}^{\infty} f(\zeta) \mathrm{e}^{-i \omega \zeta} \mathrm{d} \zeta\right] \mathrm{d} y \\
\mathcal{F}((f \star g) ; \omega)=\hat{f}(\omega) \int_{-\infty}^{\infty} g(y) \mathrm{e}^{-i \omega y} \mathrm{~d} y \\
\mathcal{F}((f \star g) ; \omega)=\hat{f}(\omega) \hat{g}(\omega) .
\end{gathered}
$$

Similarly,

$$
\begin{gathered}
\mathcal{F}(f g ; \omega)=\mathcal{F}(f(x ; \omega)) \star \mathcal{F}(g(x ; \omega)) \\
\mathcal{F}(f g ; \omega)=\int_{-\infty}^{\infty} f(x) \mathrm{e}^{-i \omega x} \mathrm{~d} x \star \int_{-\infty}^{\infty} g(x) \mathrm{e}^{-i \omega x} \mathrm{~d} x \\
\mathcal{F}(f g ; \omega)=\hat{f}(\omega) \star \hat{g}(\omega) .
\end{gathered}
$$

The notion of Fourier transform is related to characteristic function in the following sense.

Definition 3: Suppose $P(x)$ is a probability distribution function with $x \in R$. Then a characteristic function $\phi(\omega), \omega \in I R$ is defined as the Fourier transform of $P(x)$ by

$$
\mathcal{F}(P(x) ; \omega) \equiv \hat{\phi}(\omega) \equiv \int_{-\infty}^{\infty} P(x) \mathrm{e}^{i \omega x} \mathrm{~d} \omega=\mathbb{E}\left[\mathrm{e}^{i \omega x}\right]
$$

One can recover the probability distribution function $P(x)$ by taking the inverse Fourier transform of the characteristics function just as the definition of inverse Fourier transform presented in Equation (2).

This is given by

$$
P(x)=\mathcal{F}^{-1}(\hat{\phi}(\omega))=\frac{1}{2 \pi} \int_{-\infty}^{\infty} \hat{\phi}(\omega) \mathrm{e}^{-i \omega x} \mathrm{~d} \omega
$$

The reader can see the reference ([10]) and other standard texts on Fourier transform for more information.

There are available models for predicting economic recession probabilities especially on US recession (See [11]). The application of probability theory is found useful in such estimation [12]. Philip et al. [13] gave an intuition in predicting price dynamics in the presence of economic recession via recurrence relation technique and further presented a simple model showing the future of an economy under the exposure of recession if strong strategies and policies were not put in place to revive the economy. Fabio, Fornari and Antonio, Mele in (2009) [14] showed some connections between Financial Volatility and Economic Activity.

In sequel, we examine uncertainty on options value pose by recession on the volatility parameter of an option.

Assumption: Supposed that there are two sources of volatility variation on the assets. These are classified into economic recession induced volatility, $v^{\text {rec }}$, 
and the usual volatility from other sources, $v^{*}$, all assumed to be stochastic in nature. The volatility of the options is then given by

$$
V(t)= \begin{cases}v^{r e c}+v^{*}, & \text { if the economy is in recession; } \\ v^{*}, & \text { if economy is recession free. }\end{cases}
$$

where $v^{\text {rec }}$ is economy recession induced volatility in the stock market which arises due to information flow from the recessed Economy while $v^{*}$ is the volatility in the market from other sources. The study of parameters uncertainties in some financial models has received attention in the past few years. Two of those authors are [15] and [16]. Unfortunantely, many authors are yet to consider uncertaianties parameters that focus on economic recession in most Financial models use especially in derivative pricing. This present research is believed to stand out in that regards as it is hoped that more attention of authors will be drawn to economic recession and its effects in options pricing.

\section{The Main Result}

1) The Fast Fourier Transform (FFT) algorithm goes thus.

2) First get the characteristic function of the distribution. 2. Derive an analytic expression for the Fourier Transform of the modified options price wrt the characteristic function.

3) Find the Fourier inversion of the pricing function.

4) Perform Discrete Fourier transform in both step 2 and 3 above and apply Trapezoidal rule.

5) Choose uniform grid size for the computation of the FFT in step 4.

6) Make choices on the values assumed by other parameters such as $N$, the decay rate $(\alpha)$, etc. for optimality purpose.

\subsection{Fast Fourier Transform of Multi-Assets in Finite Dimension}

Consider an $n$-factor underlying asset $S_{j}, j=1,2, \cdots, n$. The payoff function $H(S, K)$ of the options at maturity time on the given underlying multi-assets is defined as

$$
\begin{gathered}
H(S, K)=\prod_{j=1}^{n}\left(c\left(S_{j}-K_{j}\right)^{+}\right) . \\
H(S, K)=\left(c\left(S_{1}-K_{1}\right)^{+} \cdot c\left(S_{2}-K_{2}\right)^{+} \cdot c\left(S_{3}-K_{3}\right)^{+} \cdots c\left(S_{n}-K_{n}\right)^{+}\right)
\end{gathered}
$$

where the $S_{j}$ and $K_{j}$ are respectively asset and strike prices.

The

$$
\left(S_{j}-K_{j}\right)^{+}=\left(\max \left(S_{j}-K_{j}, 0\right), \text { for each } j=1,2, \cdots, n\right)
$$

The parameter $c$ is a control switch between call or put options. If $c$ as defined in the Equations (19) and (20) is -1 , then one has put option and if $c=+1$, one has call option.

Let $k_{j}, s_{j} \mid j=1,2, \cdots, n$ be logarithm of the strikes $K_{j}$ and assets $S_{j}$ prices 
respectively. Then, the option values computation in $n$-dimension is given by

$$
\begin{aligned}
& V_{T}\left(k_{1}, k_{2}, \cdots, k_{n}\right) \\
& =E^{\mathcal{Q}}\left(\mathrm{e}^{-r T}\left(S_{1}(T)-K_{1}(T)\right)^{+} \cdot\left(S_{2}(T)-K_{2}(T)\right)^{+} \cdots\left(S_{n}(T)-K_{n}(T)\right)^{+}\right)
\end{aligned}
$$

where $\mathcal{Q}$ is taken to be risk neutral measure while $q_{T}$ is the joint density function of each $s_{j}(T), j=1,2, \cdots, n$.

The integral representation of Equation (21) is given as

$$
\begin{aligned}
& V_{T}\left(k_{1}, k_{2}, \cdots, k_{n}\right) \\
& =\int_{k_{1}}^{\infty} \int_{k_{2}}^{\infty} \cdots \int_{k_{n}}^{\infty} \mathrm{e}^{-r T}\left(\mathrm{e}^{s_{1}}-\mathrm{e}^{k_{1}}\right)\left(\mathrm{e}^{s_{2}}-\mathrm{e}^{k_{2}}\right) \cdots\left(\mathrm{e}^{s_{n}}-\mathrm{e}^{k_{n}}\right) \\
& \quad \times q_{T}\left(s_{1}, s_{2}, \cdots, s_{n}\right) \mathrm{d} s_{n} \cdots \mathrm{d} s_{2} \mathrm{ds}_{1}
\end{aligned}
$$

We gave the characteristic function corresponding to the joint density as

$$
\begin{gathered}
\varphi\left(u_{1}, u_{2}, \cdots, u_{n}\right)=E^{\mathcal{Q}}\left(\mathrm{e}^{\sum i u_{j} s_{j}(T)}\right) \\
\varphi\left(u_{1}, u_{2}, \cdots, u_{n}\right)=\int_{-\infty}^{\infty} \mathrm{e}^{i u_{1} s_{1}+i u_{2} s_{2}+\cdots+i u_{n} s_{n}} q_{T}\left(s_{1}, s_{2}, \cdots, s_{n}\right) \mathrm{d} s_{n} \cdots \mathrm{d} s_{2} \mathrm{~d} s_{1} .
\end{gathered}
$$

In order to ensure the square integrability of the right hand side of the Equation (22), we take its product and a decaying term $\alpha$ over $k_{j}$.

This we expressed as

$$
c_{T}\left(k_{1} k_{2}, \cdots, k_{n}\right)=\mathrm{e}^{\alpha_{1} k_{1}+\alpha_{2} k_{2}+\cdots+\alpha_{n} k_{n}} \times V_{T}\left(k_{1}, k_{2}, \cdots, k_{n}\right), \alpha_{1}, \alpha_{2}, \cdots, \alpha_{n}>0 .
$$

For the call option, the Fourier transform of the modified option prices is given by

$$
\begin{aligned}
& \psi_{T}\left(u_{1}, u_{2}, \cdots, u_{n}\right) \\
& =\int_{-\infty}^{\infty} \int_{-\infty}^{\infty} \cdots \int_{-\infty}^{\infty} \mathrm{e}^{i\left(u_{1} k_{1}+u_{2} k_{2}+\cdots+u_{n} k_{n}\right)} C_{T}\left(k_{1}, k_{2}, \cdots, k_{n}\right) \mathrm{d} k_{n} \cdots \mathrm{d} k_{2} \mathrm{~d} k_{1} \\
& \quad \psi_{T}\left(u_{1}, u_{2}, \cdots, u_{n}\right) \\
& =\int \cdots \int_{R^{n}}\left(\mathrm{e}^{\left(\alpha_{1}+u_{1}\right) k_{1}+\left(\alpha_{2}+u_{u_{2}}\right) k_{2}+\cdots+\left(\alpha_{n}+i u_{n}\right) k_{n}}\right. \\
& \quad \times \int_{k_{n}}^{+\infty} \cdots \int_{k_{2}}^{+\infty} \int_{k_{1}}^{+\infty} \mathrm{e}^{-r T}\left(\mathrm{e}^{s_{1}}-\mathrm{e}^{k_{1}}\right)\left(\mathrm{e}^{s_{2}}-\mathrm{e}^{k_{2}}\right) \cdots\left(\mathrm{e}^{s_{n}}-\mathrm{e}^{k_{n}}\right) \\
& \quad \times q_{T}\left(s_{1}, s_{2}, \cdots, s_{n}\right) \mathrm{d} k_{n} \cdots \mathrm{d} k_{2} \mathrm{~d} k_{1} \mathrm{~d} s_{n} \cdots \mathrm{d} s_{2} \mathrm{~d} s_{1}
\end{aligned}
$$

This is further simplified as follows

$$
\begin{aligned}
& \psi_{T}\left(u_{1}, u_{2}, \cdots, u_{n}\right) \\
& =\int \cdots \int_{R^{n}} \mathrm{e}^{-r T} q_{T}\left(s_{1}, s_{2}, \cdots, s_{n}\right) \\
& \quad \times \int_{\infty}^{s^{n}} \cdots \int_{\infty}^{s^{2}} \int_{\infty}^{s^{1}} \mathrm{e}^{-r T}\left(\left(\mathrm{e}^{s_{1}}-\mathrm{e}^{k_{1}}\right)\left(\mathrm{e}^{s_{2}}-\mathrm{e}^{k_{2}}\right) \cdots\left(\mathrm{e}^{s_{n}}-\mathrm{e}^{k_{n}}\right)\right) \\
& \quad \times \mathrm{e}^{\left(\alpha_{1}+i u_{1}\right) k_{1}+\left(\alpha_{2}+i u_{2}\right) k_{2}+\cdots+\left(\alpha_{n}+i u_{n}\right) k_{n}} \mathrm{~d} k_{n} \cdots \mathrm{d} k_{2} \mathrm{~d} k_{1} \mathrm{~d} s_{n} \cdots \mathrm{d} s_{2} \mathrm{~d} s_{1}
\end{aligned}
$$

which reduces to

$$
\begin{aligned}
& \psi_{T}\left(u_{1}, u_{2}, \cdots, u_{n}\right) \\
& =\int \cdots \int_{R^{n}} \mathrm{e}^{-r T} q_{T}\left(s_{1}, s_{2}, \cdots, s_{n}\right) \\
& \times\left(\frac{\left(\mathrm{e}^{\left(1+\alpha_{1}+i_{1}\right) s_{1}+\left(1+\alpha_{2}+i u_{2}\right) s_{2}+\cdots+\left(1+\alpha_{n}+i u_{n}\right) s_{n}}\right)}{\left(\alpha_{1}+i u_{1}\right)\left(1+\alpha_{1}+i u_{1}\right)\left(\alpha_{2}+i u_{2}\right)\left(1+\alpha_{2}+i u_{2}\right) \cdots\left(\alpha_{n}+i u_{n}\right)\left(1+\alpha_{n}+i u_{n}\right)}\right) \mathrm{d} s_{n} \cdots \mathrm{d} s_{2} \mathrm{~d} s_{1}
\end{aligned}
$$


For a known characteristic function or a calculated affine characteristic function $\varphi_{T}$ of a given process, it is possible to derive an analytic close form formula for $\Psi_{T}\left(u_{1}, u_{2}, \cdots, u_{n}\right)$ which is the Fourier transform of the multi-assets.

Applying definition (2), we expressed the Fourier transform of (21) through (28)-(29) as

$$
\begin{aligned}
& V_{T}\left(k_{1}, k_{2}, \cdots, k_{n}\right) \\
& =\frac{\mathrm{e}^{-\left(\alpha_{1} k_{1}+\alpha_{2} k_{2}+\cdots+\alpha_{n} k_{n}\right)}}{(2 \pi)^{n}} \underbrace{\int_{-\infty}^{\infty} \cdots \int_{-\infty}^{\infty}}_{n \text {-tuple }} \mathrm{e}^{-i\left(u_{1} k_{1}+u_{2} k_{2}+\cdots+u_{n} k_{n}\right)} \psi_{T}\left(u_{1}, u_{2}, \cdots, u_{n}\right) \mathrm{d} u_{k} \cdots \mathrm{d} u_{2} \mathrm{~d} u_{1} .
\end{aligned}
$$

Rewriting the integrals in series form using trapezoidal rule, equation (30) is transformed to

$$
\begin{aligned}
& V_{T}\left(k_{1}, k_{2}, \cdots, k_{n}\right) \\
& =\frac{\mathrm{e}^{-\left(\alpha_{1} k_{1}+\alpha_{2} k_{2}+\cdots+\alpha_{n} k_{n}\right)}}{(2 \pi)^{n}} \underbrace{\sum_{n \text {-tuple }}^{\sum_{m_{1}=1}^{N_{1}} \uparrow \sum_{m_{2}=1}^{N_{2}} \uparrow \cdots \sum_{m_{n}=1}^{N_{n}} \uparrow \mathrm{e}^{-i\left(u_{1, m_{1}} k_{1}+u_{2, m_{2}} k_{2}\right)} \psi_{T}\left(u_{1, m_{1}}, u_{2, m_{2}}, \cdots, u_{n, m_{n}}\right)} \prod_{j=1}^{n} h_{j}}_{\Omega\left(k_{1}, k_{2}, \cdots, k_{n}\right)}
\end{aligned}
$$

where

$$
\prod^{n}\left\lceil h_{j}=h_{1} h_{2} \cdots h_{n} \text {, while } h_{j}, j=1,2, \cdots, n\right. \text { denote integration steps here }
$$

For a single asset, Fast Fourier Transform (FFT) equation is defined in the form of

$$
H(k)=\sum_{j=1}^{N-1} \mathrm{e}^{-i \frac{2 \pi}{N}(j-1)(k-1)} y_{j}, 1 \leq k \leq N .
$$

Therefore, in the light of Equation (32), we gave an $n$-dimension version of $y_{j}, j=1,2, \cdots, n$, such that in an effort to capture the imaginary part of the complex form of $y_{j}$, which is inclusive in Fourier transform, we have

$$
\xi_{j} \mid j=1,2, \cdots, n:=\sum_{m_{1}=1}^{N_{1}-1} \sum_{m_{2}=1}^{N_{1}-1} \cdots \sum_{m_{n}=1}^{N_{1}-1} \mathrm{e}^{-i\left(\frac{2 \pi}{N_{1}} j_{1} m_{1}+\frac{2 \pi}{N_{2}} j_{2} m_{2}+\cdots+\frac{2 \pi}{N_{n}} j_{n} m_{n}\right)} y_{j}, j=1,2, \cdots, n
$$

where $m_{1}=1,2, \cdots, N_{1}-1 ; m_{2}=1,2, \cdots, N_{2}-1$ respectively.

The integration steps $h_{j}, j=1,2, \cdots, n$, in (31) is uniformly chosen such that

$$
h_{1}=\frac{u_{1, m_{1}}}{\left(m_{1}-\frac{N}{2}\right)}, h_{2}=\frac{u_{2, m_{2}}}{\left(m_{2}-\frac{N}{2}\right)}, \cdots, h_{n}=\frac{u_{n, m_{n}}}{\left(m_{n}-\frac{N}{2}\right)}
$$

Considering a uniform grid size of $N \times N$ array and set.

The size of the uniform spacing $\zeta$ between the $N$ values of vector $k$ with respect to the integration steps $h_{j}, j=1,2, \cdots, n$ and Equation (33) has the following relation

$$
\zeta_{1} h_{1}=\zeta_{2} h_{2}=\cdots=\zeta_{n} h_{n}=\frac{2 \pi}{N}
$$

The options prices $V_{T}$ over log strikes from Equation (31) is now given as 


$$
\begin{aligned}
& V_{T}\left(k_{1, p_{1}}, k_{2, p_{2}}, \cdots, k_{n, p_{n}}\right) \\
& \approx \frac{\mathrm{e}^{-\left(\alpha_{1} k_{1, p_{1}}+\alpha_{2} k_{2, p_{2}}+\cdots+\alpha_{n} k_{n, p_{n}}\right)}}{(2 \pi)^{n}} \Omega\left(k_{1, p_{1}}, k_{2, p_{2}}, \cdots, k_{n, p_{n}}\right) \prod_{j=1}^{n} h_{j}, \\
& \text { for } 0 \leq p_{1}, p_{2}, \cdots, p_{n} \leq N-1 .
\end{aligned}
$$

where

$$
\begin{aligned}
& \Omega\left(k_{1, p_{1}}, k_{2, p_{2}}, \cdots, k_{n, p_{n}}\right) \\
& =\sum_{m_{1}=1}^{N_{1}-1} \sum_{m_{2}=1}^{N_{1}-1} \cdots \sum_{m_{n}=1}^{N_{1}-1} \mathrm{e}^{-\frac{2 \pi}{N}\left(\left(m_{1}-\frac{N}{2}\right)\left(p_{1}-\frac{N}{2}\right)+\left(m_{2}-\frac{N}{2}\right)\left(p_{2}-\frac{N}{2}\right)+\cdots+\left(m_{n}-\frac{N}{2}\right)\left(p_{n}-\frac{N}{2}\right)\right)} \psi_{T}\left(u_{1}, u_{2}, \cdots, u_{n}\right)
\end{aligned}
$$

\section{Remarks:}

1) The Equation (35) above is fast Fourier transform formula which can be adopted to calculate the value of European call option prices on finite $n$-dimensional underlying multi-assets.

2) The corresponding European put option value can be obtained if we set the control parameter $c=-1$ in the Equations (19)-(20) such that the sign attached to the log strikes and log assets changes in Equation (21) follow the same procedure through to arrive at (35). Alternatively, the put values can be obtained via put-call parity.

\subsection{Fast Fourier Transform of Multi-Assets in an Infinite Dimension}

Following similar algorithm presented in finite dimensional case, the above formulation can be extended to an infinite dimension as follows.

The payoff function $H(S, K)$ of the options at maturity time on the given underlying multi-assets in an infinite dimension is given as

$$
H(S, K)=\prod_{j=1}^{\infty}\left(c\left(S_{j}-K_{j}\right)\right)
$$

$$
\begin{aligned}
& H(S, K) \\
& =\left(c\left(S_{1}-K_{1}\right)^{+} \cdot c\left(S_{2}-K_{2}\right)^{+} \cdot c\left(S_{3}-K_{3}\right)^{+} \cdots c\left(S_{n}-K_{n}\right)^{+} \cdot c\left(S_{n+1}-K_{n+1}\right)^{+} \cdots\right)
\end{aligned}
$$

where the $S_{j}$ and $K_{j}$ are respectively asset and strike prices. The parameter $c$ is a control in switching between call or put options as discussed earlier.

Let $k_{j}, s_{j} \mid j=1,2, \cdots, n, n+1, \cdots$ be logarithm of the strikes $K_{j}$ and assets $S_{j}$ prices respectively. Then, the option values computation in infinite-dimension is given by

$$
\begin{aligned}
& V_{T}\left(k_{1}, k_{2}, \cdots, k_{n+1}, \cdots\right) \\
& =E^{\mathcal{Q}}\left(\mathrm{e}^{-r T}\left(S_{1_{T}}-K_{1_{T}}\right)^{+} \cdot\left(S_{2_{T}}-K_{2_{T}}\right)^{+} \cdots\left(S_{n_{T}}-K_{n_{T}}\right)^{+} \cdot\left(S_{n+1_{T}}-K_{n+1_{T}}\right)^{+} \cdots\right)
\end{aligned}
$$

where $\mathcal{Q}$ still remain as risk neutral measure and $q_{T}$ is the joint density function for each

$$
s_{j}(T), j=1,2, \cdots, n, n+1, \cdots
$$


The characteristic function corresponding to the joint density is given as

$$
\begin{gathered}
\varphi\left(u_{1}, u_{2}, \cdots, u_{n+1}, \cdots\right)=E^{\mathcal{Q}}\left(\mathrm{e}^{\sum \tilde{i} u_{j} s_{j}(T)}\right) \\
\varphi\left(u_{1}, u_{2}, \cdots, u_{n+1}, \cdots\right) \\
=\int_{-\infty}^{\infty} \mathrm{e}^{i u_{1} s_{1}+i u_{2} s_{2}+\cdots+i u_{n+1} s_{n+1}+\cdots} q_{T}\left(s_{1}, s_{2}, \cdots, s_{n+1}, \cdots\right) \mathrm{d} s_{n+1} \cdots \mathrm{d} s_{2} \mathrm{~d} s_{1} .
\end{gathered}
$$

The Fourier transform of the modified call option prices is given by

$$
\begin{aligned}
& \varphi\left(u_{1}, u_{2}, \cdots, u_{n+1}, \cdots\right) \\
& =\int_{-\infty}^{\infty} \int_{-\infty}^{\infty} \cdots \int_{-\infty}^{\infty} \mathrm{e}^{i\left(u_{1} k_{1}+u_{2} k_{2}+\cdots+u_{n+1} k_{n+1}+\cdots\right)} c_{T}\left(k_{1}, k_{2}, \cdots, k_{n+1}, \cdots\right) \mathrm{d} k_{n+1} \cdots \mathrm{d} k_{2} \mathrm{~d} k_{1} \\
& \psi_{T}\left(u_{1}, u_{2}, \cdots, u_{n+1}, \cdots\right) \\
& =\int \cdots \int_{I R^{n}} \mathrm{e}^{\left(\alpha_{1}+i u_{1}\right) k_{1}+\left(\alpha_{2}+i u_{2}\right) k_{2}+\cdots+\left(\alpha_{n+1}+i u_{n+1}\right) k_{n+1}+\cdots} \\
& \quad \times \int_{k_{n+1}}^{+\infty} \cdots \int_{k_{2}}^{+\infty} \int_{k_{1}}^{+\infty} \mathrm{e}^{-r T}\left(\left(\mathrm{e}^{s_{1}}-\mathrm{e}^{k_{1}}\right)\left(\mathrm{e}^{s_{2}}-\mathrm{e}^{k_{2}}\right) \cdots\left(\mathrm{e}^{s_{n+1}}-\mathrm{e}^{k_{n+1}}\right) \cdots\right) \\
& \quad \times q_{T}\left(s_{1}, s_{2}, \cdots, s_{n+1}, \cdots\right) \mathrm{d} k_{n+1} \cdots \mathrm{d} k_{2} \mathrm{~d} k_{1} \mathrm{~d} s_{n+1} \cdots \mathrm{d} s_{2} \mathrm{~d} s_{1}
\end{aligned}
$$

Further simplification yields

$$
\begin{aligned}
& \psi_{T}\left(u_{1}, u_{2}, \cdots, u_{n+1}, \cdots\right) \\
& =\int \cdots \int \mathrm{e}^{-r T} q_{T}\left(s_{1}, s_{2}, \cdots, s_{n+1}, \cdots\right) \\
& \quad \times \int_{\infty}^{s^{2}} \int_{\infty}^{s^{1}} \mathrm{e}^{-r T}\left(\left(\mathrm{e}^{s_{1}}-\mathrm{e}^{k_{1}}\right)\left(\mathrm{e}^{s_{2}}-\mathrm{e}^{k_{2}}\right) \cdots\left(\mathrm{e}^{s_{n+1}}-\mathrm{e}^{k_{n+1}}\right) \cdots\right) \\
& \quad \times \mathrm{e}^{\left(\alpha_{1}+i u_{1}\right) k_{1}+\left(\alpha_{2}+i u_{2}\right) k_{2}+\cdots+\left(\alpha_{n+1}+i u_{n+1}\right) k_{n+1}+\cdots} \mathrm{d} k_{n+1} \cdots \mathrm{d} k_{2} \mathrm{~d} k_{1} \mathrm{~d} s_{n+1} \cdots \mathrm{d} s_{2} \mathrm{ds} s_{1}
\end{aligned}
$$

which reduces to

$$
\begin{aligned}
& \psi_{T}\left(u_{1}, u_{2}, \cdots, u_{n+1}, \cdots\right) \\
& =\int \cdots \int_{R^{\infty}} \mathrm{e}^{-r T} q_{T}\left(s_{1}, s_{2}, \cdots, s_{n+1}, \cdots\right) \\
& \times\left(\frac{\left(\mathrm{e}^{\left(1+\alpha_{1}+i u_{1}\right) s_{1}+\left(1+\alpha_{2}+i u_{2}\right) s_{2}+\cdots+\left(1+\alpha_{n+1}+i u_{n+1}\right) s_{n+1}+\cdots}\right)}{\left(\alpha_{1}+i u_{1}\right)\left(1+\alpha_{1}+i u_{1}\right)\left(\alpha_{2}+i u_{2}\right)\left(1+\alpha_{2}+i u_{2}\right) \cdots\left(\alpha_{n+1}+i u_{n+1}\right)\left(1+\alpha_{n+1}+i u_{n+1}\right) \cdots}\right) \cdots \mathrm{d} s_{n+1} \cdots \mathrm{d} s_{2} \mathrm{~d} s_{1}
\end{aligned}
$$

Expressing the integrals in discrete Fourier transform and using trapezoidal rule, we have

$$
\begin{aligned}
& V_{T}\left(k_{1}, k_{2}, \cdots, k_{n+1}, \cdots\right)=\frac{\mathrm{e}^{-\left(\alpha_{1} k_{1}+\alpha_{2} k_{2}+\cdots+\alpha_{n+1} k_{n+1}+\cdots\right)}}{(2 \pi)^{n+v}} \\
& \times \sum_{m_{1}=1}^{N_{1}} \sum_{m_{2}=1}^{N_{2}} \cdots \sum_{m_{\infty}}^{N_{\infty}} \mathrm{e}^{-i\left(u_{1, m_{1}} k_{1}+u_{2, m_{2}} k_{2}+\cdots\right)} \psi_{T}\left(u_{1, m_{1}}, u_{2, m_{2}}, \cdots, u_{n+1, m_{n+1}} \cdots\right) \prod_{j=1}^{\infty} h_{j}
\end{aligned}
$$

where

$\prod^{\infty} h_{j}=h_{1} h_{2} \cdots h_{n+1} \cdots$, while $h_{j}, j=1,2, \cdots, n+1, \cdots$ denote integration steps here and $v$ is a very large integer.

The options prices $V_{T}$ over $\log$ strikes $k=\log K$ are finally given as

$$
\begin{aligned}
& V_{T}\left(k_{1, p_{1}}, k_{2, p_{2}} \cdots, k_{n+1, p_{n+1}}, \cdots\right) \\
& \approx \frac{\mathrm{e}^{-\left(\alpha_{1} k_{1, p_{1}}+\alpha_{2} k_{2, p_{2}}+\cdots+\alpha_{n+1} k_{n+1, p_{n+1}}+\cdots\right)}}{(2 \pi)^{n+v}} \Omega\left(k_{1, p_{1}}, k_{2, p_{2}}, \cdots, k_{n, p_{n}}\right) \prod_{j=1}^{\infty} h_{j}
\end{aligned}
$$


where $0 \leq p_{1}, p_{2}, \cdots, p_{n+1}, \cdots \leq N-1$ and

$$
\begin{aligned}
& \Omega\left(k_{1, p_{1}}, k_{2, p_{2}}, \cdots, k_{n+1, p_{n+1}}, \cdots\right) \\
& =\sum_{m_{1}=1}^{N_{1}-1} \sum_{m_{2}=1}^{N_{1}-1} \cdots \sum_{m_{n+1}=1}^{N_{1}-1} \cdots \mathrm{e}^{-\frac{2 \pi}{N}\left(\left(m_{1}-\frac{N}{2}\right)\left(p_{1}-\frac{N}{2}\right)+\left(m_{2}-\frac{N}{2}\right)\left(p_{2}-\frac{N}{2}\right)+\cdots+\left(m_{n+1}-\frac{N}{2}\right)\left(p_{n+1}-\frac{N}{2}\right)\right)} \\
& \quad \times \psi_{T}\left(u_{1}, u_{2}, \cdots, u_{n+1} \cdots\right)
\end{aligned}
$$

We acknowledge Carr \& Madan (1999) [6] for providing a fast Fourier Transform framework on single underlying asset which we extended to multi-assets in both finite dimension and infinite dimension in this paper.

\section{Application on Three Assets in 3-Dimensional Case}

Let $S_{j} \mid j=1,2,3$ be three underlying assets and strike prices $K_{j} \mid j=1,2,3$ respectively. The payoff function is defined in the light of Equation (20) as

$H(S, K)=\left(c\left(S_{1}-K_{1}\right)^{+} \cdot c\left(S_{2}-K_{2}\right)^{+} \cdot c\left(S_{3}-K_{3}\right)^{+}\right), c=+1$ for call option. (45) where the $S_{j}$ and $K_{j}$ are respectively asset and strike prices.

The option values computation in 3-dimension is given by

$$
\begin{aligned}
& V_{T}\left(k_{1}, k_{2}, k_{3}\right) \\
& =E^{\mathcal{Q}}\left(\mathrm{e}^{-r T}\left(S_{1}(T)-K_{1}(T)\right)^{+} \cdot\left(S_{2}(T)-K_{2}(T)\right)^{+} \cdot\left(S_{3}(T)-K_{3}(T)\right)^{+}\right)
\end{aligned}
$$

where $\mathcal{Q}$ is a risk neutral measure and $q_{T}$ is the joint density function of each $s_{j}(T), j=1,2,3$.

The integral representation of Equation (46) is given as

$$
\begin{aligned}
& V_{T}\left(k_{1}, k_{2}, k_{3}\right) \\
& =\int_{k_{1}}^{\infty} \int_{k_{2}}^{\infty} \int_{k_{3}}^{\infty} \mathrm{e}^{-r T}\left(\mathrm{e}^{s_{1}}-\mathrm{e}^{k_{1}}\right)\left(\mathrm{e}^{s_{2}}-\mathrm{e}^{k_{2}}\right)\left(\mathrm{e}^{s_{3}}-\mathrm{e}^{k_{3}}\right) q_{T}\left(s_{1}, s_{2}, s_{3}\right) \mathrm{d} s_{3} \mathrm{~d} s_{2} \mathrm{~d} s_{1} .
\end{aligned}
$$

The characteristic function corresponding to the joint density is given by

$$
\begin{gathered}
\varphi\left(u_{1}, u_{2}, u_{3}\right)=E^{\mathcal{Q}}\left(\mathrm{e}^{\sum i u_{j} s_{j}(T)}\right), j=1,2,3 . \\
\varphi\left(u_{1}, u_{2}, u_{3}\right)=\int_{-\infty}^{\infty} \mathrm{e}^{i u_{1} s_{1}+i u_{2} s_{2}+i u_{3} s_{3}} q_{T}\left(s_{1}, s_{2}, s_{3}\right) \mathrm{d} s_{3} \mathrm{~d} s_{2} \mathrm{~d} s_{1} .
\end{gathered}
$$

Multiplying the right side of Equation (47) and decaying term say $\alpha$ over $k_{1}, k_{2}, k_{3}$ so as to have square integrable of the right side of (47).

We expressed the result as

$$
c_{T}\left(k_{1}, k_{2}, k_{3}\right)=\mathrm{e}^{\alpha_{1} k_{1}+\alpha_{2} k_{2}+\alpha_{3} k_{3}} \times V_{T}\left(k_{1}, k_{2}, k_{3}\right), \alpha_{1}, \alpha_{2}, \alpha_{3}>0 .
$$

For the call option, the Fourier transform of the modified option prices is given by

$$
\begin{aligned}
\psi_{T}\left(u_{1}, u_{2}, u_{3}\right)= & \int_{-\infty}^{\infty} \int_{-\infty}^{\infty} \int_{-\infty}^{\infty} \mathrm{e}^{i\left(u_{1} k_{1}+u_{2} k_{2}+u_{3} k_{3}\right)} c_{T}\left(k_{1}, k_{2}, k_{3}\right) \mathrm{d} k_{3} \mathrm{~d} k_{2} \mathrm{~d} k_{1} \\
\psi_{T}\left(u_{1}, u_{2}, u_{3}\right)= & \int_{I R^{3}} \mathrm{e}^{\left(\alpha_{1}+i u_{1}\right) k_{1}+\left(\alpha_{2}+i u_{2}\right) k_{2}+\left(\alpha_{3}+i u_{3}\right) k_{3}} \\
& \times \int_{k_{3}}^{+\infty} \int_{k_{2}}^{+\infty} \int_{k_{1}}^{+\infty} \mathrm{e}^{-r T}\left(\mathrm{e}^{s_{1}}-\mathrm{e}^{k_{1}}\right)\left(\mathrm{e}^{s_{2}}-\mathrm{e}^{k_{2}}\right)\left(\mathrm{e}^{s_{3}}-\mathrm{e}^{k_{3}}\right) \\
& \times q_{T}\left(s_{1}, s_{2}, s_{3}\right) \mathrm{d} k_{3} \mathrm{~d} k_{2} \mathrm{dk}_{1} \mathrm{~d} s_{3} \mathrm{~d} s_{2} \mathrm{~d} s_{1}
\end{aligned}
$$


Further simplification yields

$$
\begin{aligned}
\psi_{T}\left(u_{1}, u_{2}, u_{3}\right)= & \int_{I R^{3}} \mathrm{e}^{-r T} q_{T}\left(s_{1}, s_{2}, s_{3}\right) \\
& \times \int_{\infty}^{s^{3}} \int_{\infty}^{s^{2}} \int_{\infty}^{s^{1}} \mathrm{e}^{-r T}\left(\left(\mathrm{e}^{s_{1}}-\mathrm{e}^{k_{1}}\right)\left(\mathrm{e}^{s_{2}}-\mathrm{e}^{k_{2}}\right)\left(\mathrm{e}^{s_{3}}-\mathrm{e}^{k_{3}}\right)\right) \\
& \times \mathrm{e}^{\left(\alpha_{1}+i u_{1}\right) k_{1}+\left(\alpha_{2}+i u_{2}\right) k_{2}+\left(\alpha_{3}+i u_{3}\right) k_{3}} \mathrm{~d} k_{3} \mathrm{~d} k_{2} \mathrm{~d} k_{1} \mathrm{~d} s_{3} \mathrm{~d} s_{2} \mathrm{~d} s_{1}
\end{aligned}
$$

which reduces to

$$
\begin{aligned}
& \psi_{T}\left(u_{1}, u_{2}, u_{3}\right)=\int_{I R^{3}} \mathrm{e}^{-r T} q_{T}\left(s_{1}, s_{2}, s_{3}\right) \\
& \times\left[\frac{\left(\mathrm{e}^{\left(1+\alpha_{1}+i u_{1}\right) s_{1}+\left(1+\alpha_{2}+i u_{2}\right) s_{2}+\left(1+\alpha_{3}+i u_{3}\right) s_{3}}\right)}{\left(\alpha_{1}+i u_{1}\right)\left(1+\alpha_{1}+i u_{1}\right)\left(\alpha_{2}+i u_{2}\right)\left(1+\alpha_{2}+i u_{2}\right)\left(\alpha_{3}+i u_{3}\right)\left(1+\alpha_{3}+i u_{3}\right)}\right] \mathrm{d} s_{3} \mathrm{~d} s_{2} \mathrm{~d} s_{1}
\end{aligned}
$$

Consider a known characteristic function $\varphi_{T}$ for the distributions, an analytical close form formula for $\Psi_{T}\left(u_{1}, u_{2}, u_{3}\right)$ which is the Fourier transform of the 3-dimensional multi-assets is given by

$$
\begin{aligned}
& V_{T}\left(k_{1}, k_{2}, k_{3}\right) \\
& =\frac{\mathrm{e}^{-\left(\alpha_{1} k_{1}+\alpha_{2} k_{2}+\alpha_{3} k_{3}\right)}}{(2 \pi)^{3}} \int_{-\infty}^{\infty} \int_{-\infty}^{\infty} \int_{-\infty}^{\infty} \mathrm{e}^{-i\left(u_{1} k_{1}+u_{2} k_{2}+u_{3} k_{3}\right)} \psi_{T}\left(u_{1}, u_{2}, u_{3}\right) \mathrm{d} u_{3} \mathrm{~d} u_{2} \mathrm{~d} u_{1} .
\end{aligned}
$$

Rewriting the integrals in series form using trapezoidal rule, equation (55) is transformed to

$$
\begin{aligned}
& V_{T}\left(k_{1}, k_{2}, k_{3}\right) \\
& =\frac{\mathrm{e}^{-\left(\alpha_{1} k_{1}+\alpha_{2} k_{2}+\alpha_{3} k_{3}\right)}}{(2 \pi)^{3}} \underbrace{\sum_{m_{1}=1}^{N_{1}} \sum_{m_{2}=1}^{N_{2}} \sum_{m_{3}=1}^{N_{3}} \mathrm{e}^{-i\left(u_{1, m_{1}} k_{1}+u_{2, m_{2}} k_{2}\right)} \psi_{T}\left(u_{1, m_{1}}, u_{2, m_{2}}, u_{3, m_{3}}\right)}_{\Omega\left(k_{1}, k_{2}, k_{3}\right)} h_{3} h_{2} h_{1}
\end{aligned}
$$

where $h_{1} h_{2} h_{3}$, is the product of the integration steps.

The general form of Fast Fourier Transform (FFT) equation for a single asset has been given in the form

$$
H(k)=\sum_{j=1}^{N-1} \mathrm{e}^{-i \frac{2 \pi}{N}(j-1)(k-1)} y_{j}, 1 \leq k \leq N .
$$

Whence, 3-dimensional version of $y_{j}, j=1,2,3$, in (57) so as to capture the imaginary part of the complex form of $y_{j}$, we have

$$
\xi_{j} \mid j=1,2,3:=\sum_{m_{1}=1}^{N_{1}-1} \sum_{m_{2}=1}^{N_{1}-1} \sum_{m_{3}=1}^{N_{1}-1} \mathrm{e}^{-i\left(\frac{2 \pi}{N_{1}} j_{1} m_{1}+\frac{2 \pi}{N_{2}} j_{2} m_{2}+\frac{2 \pi}{N_{3}} j_{3} m_{3}\right)} y_{j}, j=1,2,3 .
$$

where $m_{1}=1,2, \cdots, N_{1}-1 ; m_{2}=1,2, \cdots, N_{2}-1 ; m_{3}=1,2, \cdots, N_{2}-1$, respectively.

The integration steps $h_{j}, j=1,2,3$, in (56) is uniformly chosen such that

$$
h_{1}=\frac{u_{1, m_{1}}}{\left(m_{1}-\frac{N}{2}\right)}, h_{2}=\frac{u_{2, m_{2}}}{\left(m_{2}-\frac{N}{2}\right)}, h_{3}=\frac{u_{3, m_{3}}}{\left(m_{3}-\frac{N}{2}\right)}
$$

Considering a uniform grid size of $N \times N$ array and set. 
The size of the uniform spacing $\zeta$ between the $N$ values of vector $k$ with respect to the integration steps $h_{j}, j=1,2,3$ and Equation (58) has the following relation

$$
\zeta_{1} h_{1}=\zeta_{2} h_{2}=\zeta_{3} h_{3}=\frac{2 \pi}{N}
$$

The options prices $V_{T}$ over log strikes from Equation (56) is given by

$$
\begin{aligned}
& V_{T}\left(k_{1, p_{1}}, k_{2, p_{2}}, k_{3, p_{3}}\right) \\
& \approx \frac{\mathrm{e}^{-\left(\alpha_{1} k_{1, p_{1}}+\alpha_{2} k_{2, p_{2}}+\alpha_{3} k_{3, p_{3}}\right)}}{(2 \pi)^{3}} \Omega\left(k_{1, p_{1}}, k_{2, p_{2}}, k_{3, p_{3}}\right) h_{1} h_{2} h_{3}, \\
& \text { for } 0 \leq p_{1}, p_{2}, p_{3} \leq N-1 .
\end{aligned}
$$

where

$$
\begin{aligned}
& \Omega\left(k_{1, p_{1}}, k_{2, p_{2}}, k_{3, p_{3}}\right) \\
& =\sum_{m_{1}=1}^{N_{1}-1} \sum_{m_{2}=1}^{N_{1}-1} \sum_{m_{3}=1}^{N_{1}-1} \mathrm{e}^{-\frac{2 \pi}{N}\left(\left(m_{1}-\frac{N}{2}\right)\left(p_{1}-\frac{N}{2}\right)+\left(m_{2}-\frac{N}{2}\right)\left(p_{2}-\frac{N}{2}\right)+\left(m_{3}-\frac{N}{2}\right)\left(p_{3}-\frac{N}{2}\right)\right)} \psi_{T}\left(u_{1}, u_{2}, u_{3}\right)
\end{aligned}
$$

\section{Numerical Results in 3-Dimensional Assets Case}

\subsection{Parameters Specification}

Consider call option in European sense on an underlying vector of assets and Strikes prices given as

$$
\begin{gathered}
S:=\left\{\begin{array}{l}
S_{1}=100 ; \\
S_{2}=105 ; \\
S_{3}=110
\end{array}\right. \\
K:=\left\{\begin{array}{l}
K_{1}=80 ; \\
K_{2}=85 ; \\
K_{3}=90
\end{array}\right.
\end{gathered}
$$

respectively.

Taking interest rate $r=0.04$, dividend rate $d=0.015$ and exercising time $T=\{0.5,1\}$ for all the assets. The fast Fourier Transform algorithm was used to generate the results in the table below. Setting fineness of integration grid $N=2^{12}$ and maintain the same integrability parameter $\alpha:=\left\{\alpha_{1}=\alpha_{2}=\alpha_{3}\right\}=0.25$. For illustration purpose, we assumed the volatilities are stochastic and $\operatorname{randn}()$ function was used to generate 3 array of numbers between 1 and 3 for volatilities from the two sources explained by (18) in each case. The following results in the Tables were obtained.

\subsection{Tables of Results}

Using the same data for the parameters in Table 1 except that dividend parameter is set to zero. We obtain Table 2. 
Table 1. Numerical value for a dividend paying multi-assets call options in 3-dimensions with two sources of volatility.

\begin{tabular}{ccccccc}
\hline $\begin{array}{c}\text { 3-underlying } \\
\text { Assets Prices }(S)\end{array}$ & $\begin{array}{c}\text { Strike prices } \\
(K)\end{array}$ & $\begin{array}{c}\text { Volatility } \\
\left\{\sigma^{*}, \sigma^{\text {rec }}\right\}\end{array}$ & $\begin{array}{c}\text { Exercising } \\
\text { Time }(\text { year) }\end{array}$ & $\begin{array}{c}\text { European call Options } \\
\text { Prices (FFT) }\end{array}$ & $\begin{array}{c}\text { European call Options } \\
\text { Prices (BSM) }\end{array}$ & $\begin{array}{c}\text { Output } \\
\text { Metrics }\end{array}$ \\
\hline$S_{1}=100$ & 80 & $0.93001,0.074916$ & 0.5 & 36.5090918034085 & 36.36246996 & 0.1466 \\
$S_{2}=105$ & 85 & $-1.85574,-0.098738$ & 0.5 & 58.4844133882581 & 58.84131851 & -0.3569 \\
$S_{3}=110$ & 90 & $0.89337,1.139327$ & 0.5 & 62.3503964521184 & 63.03609669 & -0.6857 \\
$S_{1}=100$ & 80 & $0.31079,-0.052239$ & 1 & 23.8042830392887 & 23.66008671 & 0.14419 \\
$S_{2}=105$ & 85 & $-1.38470,1.571944$ & 1 & 22.5677094491566 & 22.61602268 & -0.0483 \\
$S_{3}=110$ & 90 & $0.71091,-0.912938$ & 1 & 22.9463648747717 & 23.18429634 & -0.2379 \\
\hline
\end{tabular}

Table 2. Numerical value for a non-dividend paying multi-assets call options in 3-dimensions with two sources of volatility.

\begin{tabular}{ccccccc}
\hline $\begin{array}{c}\text { 3-underlying } \\
\text { Assets Prices }(S)\end{array}$ & $\begin{array}{c}\text { Strike prices } \\
(K)\end{array}$ & $\begin{array}{c}\text { Volatility } \\
\left\{\sigma^{*}, \sigma^{\text {rec }}\right\}\end{array}$ & $\begin{array}{c}\text { Exercising } \\
\text { Time }(\text { year })\end{array}$ & $\begin{array}{c}\text { European call Options } \\
\text { Prices (FFT) }\end{array}$ & $\begin{array}{c}\text { European call Options } \\
\text { Prices (BSM) }\end{array}$ & $\begin{array}{c}\text { Output } \\
\text { metrics }\end{array}$ \\
\hline$S_{1}=100$ & 80 & $0.93001,0.074916$ & 0.5 & 37.0739574519076 & 36.92704398 & 0.1469 \\
$S_{2}=105$ & 85 & $-1.85574,-0.098738$ & 0.5 & 59.1066925306292 & 59.47219301 & -0.3655 \\
$S_{3}=110$ & 90 & $0.93001,0.074916$ & 0.5 & 55.6155902567383 & 55.89405735 & -0.2784 \\
$S_{1}=100$ & 80 & $0.31079,-0.052239$ & 1 & 25.0974529765726 & 24.95267554 & 0.1448 \\
$S_{2}=105$ & 85 & $-1.38470,1.571944$ & 1 & 24.0013372611456 & 24.05195616 & -0.0506 \\
$S_{3}=110$ & 90 & $0.71091,-0.912938$ & 1 & 24.4073616767388 & 24.65044343 & -0.2431 \\
\hline
\end{tabular}

\section{Conclusion}

Fast Fourier Transform algorithm for valuation of multi-assets in both finite and infinite dimension was presented in this research work. The notion of economic recession induced volatility uncertainty was introduced in our formulation. An intuition behind the introduction of recession induced volatility uncertainty is revealed by huge volatility variation during the period of Economic recession compared to the period of normalcy (recession-free). Nigeria economic recession outbreak in 2016 and its effects on the payoffs uncertainty of Nigeria Stocks Exchange (NSE) among other invetments were among the motivating factors for proposing economic recession induced volatility formulation in Options pricing. This work generalizes the Fast Fourier transform algorithm of Carr \& Madan (2009) [6] in the valuation of European call options on a single underlying asset. The algorithm is extended further to include some other steps, then applied to value multi-assets European options in finite and infinite dimensions. Numerical experiment was presented taking Black Scholes model as a bench mark. It is believed that this research work will enhance better understanding of Fast Fourier Transform valuation of multi-assets options in multi-dimensions. We gave the Options output metrics between the two methods. The result obtained is still comparable with little errors hinged on parameters uncertainties. 


\section{Acknowledgements}

This research is partly supported by the Editorial assistants of SCIRP for helping us to apply for a discount on the article processing fees which was reduced to a considerate amount.

\section{Conflicts of Interest}

The authors declare no conflicts of interest regarding the publication of this paper.

\section{References}

[1] Black, F. and Scholes, M. (1973) The Pricing of Options and Corporate Liabilities. Journal of Political Economy, 81, 637-654. https://doi.org/10.1086/260062

[2] Kim, B.J., Ma, Y.-K. and Choe, H.J. (2013) A Simple Numerical Method for Pricing an American Put Option. Journal of Applied Mathematics, 2013, Article ID: 128025. https://doi.org/10.1155/2013/128025

[3] Chen, Y.W. (2017) Numerical Methods for Pricing Multi-Asset Options. University of Toronto, Toronto, 1-75.

[4] Alexander, C. and Venkatramanan, A. (2009) Analytic Approximations for Multi-Asset Option Pricing. ICMA Centre Discussion Papers.

[5] Horst, U. and Xia, X. (2018) Multi-Dimensional Optimal Trade Execution under Stochastic Resilience. Mathematics, arXiv:1804.03896v.

[6] Carr, P. and Madan, D.B. (1999) Option Valuation Using Fast Fourier Transform. Journal of Computational Finance, 2, 61-73. https://doi.org/10.21314/JCF.1999.043

[7] Hurdy, T.R. and Zhou, Z.W. (2009) A Fourier Transform Method for Spread Option Pricing. 1-17. arXiv:0902.3643v1 [q-fin.CP]

[8] Huang, J.X., Zhu, W.L. and Ruan, X.F. (2014) Option Pricing Using the Fast Fourier Transform under the Double Exponential Jump Model with Stochastic Volatility and Stochastic Intensity. Journal of Computational and Applied Mathematics, 263 152-159. https://doi.org/10.1016/j.cam.2013.12.009

[9] Zhylyevskyy, O. (2010) A Fast Fourier Transform Technique for Pricing American Options under Stochastic Volatility. Review of Derivatives Research, 13, 1-24. https://doi.org/10.1007/s11147-009-9041-6

[10] Sajib, B., Ruppa, K.T. and Parimala, T. (2004) Fast Fourier Transform for Option Pricing: Improved Mathematical Modeling and Design of an Efficient Parallel Algorithm. Lecture Notes in Computer Science, 3045, 686-695.

[11] Hsu, T. (2016) U.S. Recession Forecasting Using Probit Models with Asset Index Predictor Variables. 1-41.

[12] Ugbebor, O.O. (2011) Lecture Notes on Probability Theory, Stochastic Processes and Applications in Mathematical Finance. University of Ibadan, Ibadan, Nigeria.

[13] Bankole Philip, A., Ojo Ezekiel, K. and Odumosu Olukemi, M. (2017) On Recurrence Relations and Application in Predicting Price Dynamics in the Presence of Economic Recession. International Journal of Discrete Mathematics, 2, 125-131.

[14] Fornari, F. and Mele, A. (2009) Financial Volatility and Economic Activity. SSRN Electronic Journal, 1-60. https://doi.org/10.2139/ssrn.1501168

[15] Cont, R. (2006) Model Uncertainty and Its Impact on the Pricing of Derivative In- 
struments. Mathematical Finance, 16, 519-547.

https://doi.org/10.1111/j.1467-9965.2006.00281.x

[16] Slovik, P. (2011) Market Uncertainty and Market Instability. Proceedings of the IFC Conference on Initiatives to Address Data Gaps Revealed by the Financial Crisis, 34, 430-435. 